\title{
ASSESSMENT OF GROUND WATER QUALITY AT MUNICIPAL SOLID WASTE DUMPING SITE - VALAMPURIVILLAI, NAGERCOIL
}

\author{
S.Sidhardhan ${ }^{1}$, S. Adish Kumar ${ }^{2}$ \\ ${ }^{I}$ Professor, Dept. of Civil Engineering, Govt. College of Engineering, Tirunelveli, TamilNadu, India \\ ${ }^{2}$ Assistant Professor, Regional centre of Anna University, Tirunelveli, TamilNadu, India
}

\begin{abstract}
Ground water is constantly tapped for meeting rapidly growing demands of fresh water supply. Recent studies have revealed that ground water is highly susceptible to pollution from natural and anthropogenic activities. Rapid urbanization has caused numerous problems for waste disposal and in some cities these wastes are just dumped in landfills with no treatment. The leachates from these landfills contain substances that can contaminate the ground water and hence make them unsuitable to drink. The impact of such sites on the ground water quality can be assessed by monitoring the concentration of potential contaminants at a number of specific monitoring points. In this study, the quality of ground water around a municipal solid wastedumping site at Valampurivillai in Nagercoil was investigated. All the samples were tested for their physico-chemical parameters; e-coli form bacteria and heavy metal analysis. The study has shown that the ground water quality around the dumping site does not adhere and confirm to the drinking water quality standards as per Bureau of Indian Standards.
\end{abstract}

Keywords: Ground water quality, Solid Waste, Water Sampling, Heavy metal, etc...

$* * *$

\section{INTRODUCTION}

Ground water is a resource of global important, particularly in areas where access to surface is limited. Ground water resources include shallow and deep rechargeable aquifers that are connected to rivers, streams or seas while nonrenewable aquifers or fossil waters are those that may have been created by age. Ground water makes up about $95 \%$ of the world's supply of fresh water excluding fresh water in global ice.

Generally, ground water are often very poor microbiologically and may contain iron, manganese, fluoride, sulphide etc., in concentrations that exceed the standard for drinking water this is due to the composition of the ground water where the water is stored. Recently ground water contamination due to leachate generated from municipal solid waste dumpsite tends to attract the greatest public attention. If the water table is low (far below the ground surface), water will become partially filtered as it percolates downward through the soil. If the water table is high (close to the ground surface), contaminants can enter the ground water directly, without filtration by soil

Areas near landfills have a greater possibility of ground water contamination because of the potential pollution source of leachate originating from the nearby site. Such contamination of ground water resources poses a substantial risk to local resource user and to the natural environment. The impact of landfill leachate on the surface and ground water has become a major issue in solid waste management.

\section{SOLID WASTE LEACHATE}

Leaching occurs when soluble components are dissolved (leached) out of a solid material by percolating. Leachate may also carry insoluble liquids (such as oils) and small particles in the form of suspended solids. Depending on the waste types further contaminants may be introduced as a result of bio-degradation of wastes. Almost any material will produce leachate if water is allowed to percolate through it. The quality of leachate is determined primarily by the composition and solubility of the waste constituents. If waste is changing in composition (due to weathering), then leachate quality will change with time This is particularly the case in landfills containing municipal waste. The leachate intrusion depends on various factors such as location of landfill, depth of landfill, distance from landfill, temperature, and permeability of the geologic strata and direction of ground water flow.

\section{WATER SAMPLING}

The quality of sample to be collected varies with the extent of laboratory analysis to be performed. A sample volume of five litres is normally sufficient for a complete analysis. The total number of samples will depend upon the objectives of monitoring program. The use of a few strategic location and enough samples to define the results in terms of statistical significance is usually much more reliable than using many stations with only a few samples from each.

\subsection{Sampling Procedure}

Before the water samples are bottled, a minimum pumping time should be allowed in order to avoid filling of stationary well water. This time depends on the capacity and caliber of the pump. 
Samples were collected in pre-cleaned sterilized plastic cans of five liters capacity after allowing a running time of at least five minutes. The samples were brought to the laboratory within three hours and analysis was completed within 48 hours to avoid any change in physico-chemical characteristics of the water.

\subsection{Collection of Samples}

The present study was undertaken in different localities in and around Valampurivillai, Nagercoil. The residents of these localities depend on ground water for their domestic as well as their commercial requirements. Samples were taken within $2 \mathrm{~km}$ radius of the site in 8 directions .In addition to these 40 samples, one sample was collected within the premises of the municipal dumping site.

In addition to these 40 samples, one sample was collected within the premises of the municipal dumping te.

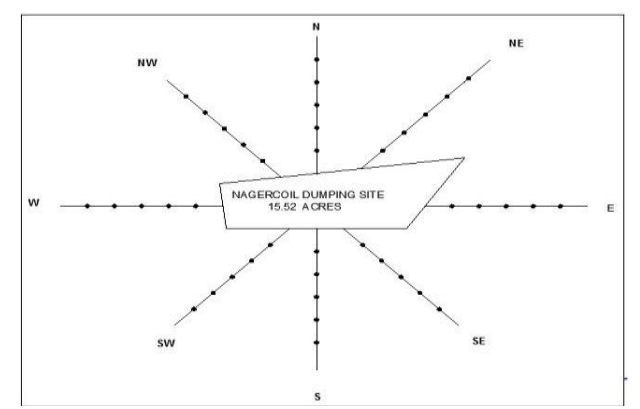

Fig.1 Sample Collection Points

\section{WATER QUALITY PARAMETERS}

The following parameters were tested for the ground water samples by confirming to BIS norms given by IS 10500 : 1991

\subsection{Physical Parameters}
1. Colour
2. Temperature
3. $\mathrm{pH}$

\subsection{Chemical parameters}
1. Acidity
2. Alkalinity
3. Chloride
4. Hardness
5. TDS
6. Sulphate
7. BOD
8. COD

\section{RESULTS}

In this study ground water analysis was free from colour and odour. The data of physic-chemical characteristic of the analyzed water samples revealed that parameters of many samples exceed the permissible limits of IS $10500: 1991$ The variation from the permissible limits is in accordance with the proximity of the sample location from the site and the upstream, downstream nature of land.

Table -1: Physic-chemical characteristic of the water samples

\begin{tabular}{|c|c|c|c|c|c|c|}
\hline \multirow[t]{2}{*}{ Parameters (mg/l) } & \multirow{2}{*}{ On-Spot } & \multicolumn{5}{|l|}{ South } \\
\hline & & S-1 & S-2 & S-3 & S-4 & S-5 \\
\hline Colour & \multicolumn{6}{|c|}{ Colourless } \\
\hline Temperature (oC) & 29.00 & 28.60 & 28.50 & 28.70 & 28.80 & 28.80 \\
\hline pH & 6.43 & 7.60 & 6.95 & 7.55 & 7.50 & 7.21 \\
\hline Acidity & 54.00 & 6.00 & 12.00 & 4.00 & 4.00 & 10.00 \\
\hline Alkalinity & 380.00 & 342.00 & 364.00 & 212.00 & 360.00 & 434.00 \\
\hline Chloride & 228.00 & 229.93 & 189.94 & 120.00 & 330.00 & 110.00 \\
\hline Hardness & 288.00 & 310.00 & 256.00 & 323.00 & 223.00 & 157.30 \\
\hline Sulphate & 47.00 & 37.34 & 47.00 & 28.00 & 0.00 & 19.00 \\
\hline BOD & 0.90 & 0.15 & 0.40 & 0.30 & 0.35 & 0.30 \\
\hline COD & 524.00 & 560.00 & 528.00 & 540.00 & 596.00 & 608.00 \\
\hline TDS (ppm) & 740.00 & 700.00 & 450.00 & 320.00 & 810.00 & 430.00 \\
\hline Lead & $<0.01$ & $<0.01$ & & & & \\
\hline Copper & 0.05 & 0.03 & & & & \\
\hline Aluminium & $<0.02$ & $<0.02$ & & & & \\
\hline Fecal Coliform & 0.00 & 0.00 & & & & \\
\hline
\end{tabular}


Table -2: Physic-chemical characteristic of the water samples

\begin{tabular}{|c|c|c|c|c|c|}
\hline \multirow[t]{2}{*}{ Parameters (mg/l) } & \multicolumn{5}{|l|}{ North } \\
\hline & N-1 & $\mathrm{N}-2$ & $\mathbf{N}-3$ & $\mathrm{~N}-4$ & $\mathrm{~N}-5$ \\
\hline Colour & \multicolumn{5}{|c|}{ Colourless } \\
\hline Temperature (oC) & 30.10 & 28.50 & 29.60 & 28.70 & 28.50 \\
\hline pH & 7.50 & 6.80 & 6.95 & 7.20 & 7.35 \\
\hline Acidity & 68.00 & 58.00 & 38.00 & 16.00 & 28.00 \\
\hline Alkalinity & 452.00 & 383.00 & 412.00 & 323.00 & 297.00 \\
\hline Chloride & 419.86 & 252.92 & 257.92 & 246.92 & 231.76 \\
\hline Hardness & 288.00 & 320.00 & 350.00 & 198.00 & 170.00 \\
\hline Sulphate & 35.20 & 48.60 & 30.30 & 27.80 & 16.50 \\
\hline BOD & 1.38 & 1.28 & 0.19 & 0.49 & 0.35 \\
\hline COD & 590.00 & 582.00 & 470.00 & 521.20 & 603.00 \\
\hline TDS (ppm) & 270.00 & 280.00 & 810.00 & 320.00 & 350.00 \\
\hline Lead & $<0.01$ & & & & \\
\hline Copper & 0.05 & & & & \\
\hline Aluminium & $<0.02$ & & & & \\
\hline
\end{tabular}

Table -3: Physic-chemical characteristic of the water samples

\begin{tabular}{|l|l|l|l|l|l|}
\hline \multirow{2}{*}{ Parameters (mg/l) } & \multicolumn{4}{l}{ North-East } \\
\cline { 2 - 6 } & NE-1 & NE-2 & NE-3 & NE-4 & NE-5 \\
\hline Colour & Colourless & \multicolumn{4}{l|}{} \\
\hline Temperature (oC) & 29.10 & 29.20 & 29.00 & 28.90 & 29.00 \\
\hline pH & 6.95 & 7.07 & 7.68 & 6.90 & 6.95 \\
\hline Acidity & 30.00 & 16.00 & 10.00 & 28.00 & 17.00 \\
\hline Alkalinity & 530.00 & 284.00 & 294.00 & 404.00 & 302.00 \\
\hline Chloride & 168.00 & 85.00 & 90.00 & 90.00 & 86.00 \\
\hline Hardness & 296.00 & 210.00 & 146.00 & 114.00 & 108.00 \\
\hline Sulphate & 93.00 & 47.00 & 28.00 & 19.00 & 16.00 \\
\hline BOD & 0.30 & 0.34 & 0.40 & 0.30 & 0.32 \\
\hline COD & 560.00 & 476.00 & 504.00 & 572.00 & 605.00 \\
\hline TDS (ppm) & 650.00 & 410.00 & 314.00 & 318.00 & 417.00 \\
\hline Lead & $<0.01$ & & & & \\
\hline Copper & 0.03 & & & & \\
\hline Aluminium & $<0.02$ & & & & \\
\hline Fecal Coliform & 0.00 & & & & \\
\hline
\end{tabular}

Table -4: Physic-chemical characteristic of the water samples 


\begin{tabular}{|l|l|l|l|l|l|} 
Temperature (oC) & 29.20 & 28.90 & 28.80 & 28.80 & 27.90 \\
\hline pH & 7.63 & 8.01 & 7.80 & 7.66 & 7.50 \\
\hline Acidity & 40.00 & 16.00 & 58.00 & 46.00 & 60.00 \\
\hline Alkalinity & 484.00 & 176.00 & 220.00 & 226.00 & 214.00 \\
\hline Chloride & 121.96 & 71.97 & 73.97 & 69.97 & 80.00 \\
\hline Hardness & 190.00 & 118.00 & 100.00 & 82.00 & 112.00 \\
\hline Sulphate & 120.00 & 198.50 & 83.30 & 57.30 & 20.00 \\
\hline BOD & 0.84 & 0.44 & 0.04 & 0.30 & 0.39 \\
\hline COD & 468.00 & 472.00 & 460.00 & 484.00 & 468.00 \\
\hline TDS (ppm) & 720.00 & 420.00 & 470.00 & 430.00 & 450.00 \\
\hline Lead & & & & & \\
\hline Copper & & & & & \\
\hline Aluminium & & & & & \\
\hline
\end{tabular}

Fecal Coliform

0.00

Table -5: Physic-chemical characteristic of the water samples

\begin{tabular}{|l|l|l|l|l|l|}
\hline \multirow{2}{*}{ Parameters (mg/I) } & \multicolumn{2}{|l}{ East } \\
\cline { 2 - 6 } & E-1 & E-2 & E-3 & E-4 & E-5 \\
\hline Colour & Colourless & \multicolumn{4}{l|}{} \\
\hline Temperature (oC) & 28.10 & 28.30 & 27.90 & 28.10 & 28.30 \\
\hline pH & 7.90 & 7.30 & 6.95 & 7.55 & 7.20 \\
\hline Acidity & 20.00 & 22.00 & 14.00 & 22.00 & 24.00 \\
\hline Alkalinity & 312.00 & 338.00 & 310.00 & 246.00 & 326.00 \\
\hline Chloride & 213.93 & 120.96 & 66.97 & 44.98 & 69.97 \\
\hline Hardness & 160.00 & 98.00 & 122.00 & 84.00 & 102.00 \\
\hline Sulphate & 28.01 & 30.56 & 37.34 & 29.72 & 32.81 \\
\hline BOD & 4.43 & 3.54 & 3.84 & 2.66 & 3.15 \\
\hline COD & 328.00 & 340.00 & 372.00 & 324.00 & 350.00 \\
\hline TDS (ppm) & 660.00 & 340.00 & 370.00 & 300.00 & 350.00 \\
\hline Lead & $<0.01$ & & & & \\
\hline Copper & 0.09 & & & & \\
\hline Aluminium & $<0.02$ & & & & \\
\hline Fecal Coliform & 0.00 & & & & \\
\hline
\end{tabular}

Table -6: Physic-chemical characteristic of the water samples

\begin{tabular}{|l|l|l|l|l|l|}
\hline \multirow{2}{*}{ Parameters (mg/I) } & West & \multicolumn{2}{l|}{} \\
\cline { 2 - 6 } & W-1 & W-2 & W-3 & W-4 & W-5 \\
\hline Colour & Colourless & \multicolumn{4}{|l|}{} \\
\hline Temperature (oC) & 28.40 & 28.10 & 28.10 & 28.00 & 28.20 \\
\hline pH & 7.50 & 6.80 & 6.95 & 7.01 & 7.80 \\
\hline Acidity & 58.00 & 18.00 & 20.00 & 16.00 & 17.00 \\
\hline Alkalinity & 128.00 & 200.00 & 270.00 & 330.00 & 294.00 \\
\hline
\end{tabular}




\begin{tabular}{|l|l|l|l|l|l|} 
Chloride & 229.92 & 76.97 & 67.97 & 84.96 & 99.50 \\
\hline Hardness & 410.00 & 198.00 & 188.00 & 126.00 & 97.80 \\
\hline Sulphate & 67.00 & 54.20 & 34.21 & 26.78 & 23.60 \\
\hline BOD & 4.63 & 4.03 & 5.32 & 3.64 & 2.63 \\
\hline COD & 376.00 & 424.00 & 520.00 & 440.00 & 392.50 \\
\hline TDS (ppm) & 1090.00 & 670.00 & 500.00 & 380.00 & 300.00 \\
\hline Lead & $<0.01$ & & & & \\
\hline Copper & $<0.03$ & & & & \\
\hline Aluminium & $<0.02$ & & & & \\
\hline
\end{tabular}

Fecal Coliform

0.00

Table -7: Physic-chemical characteristic of the water samples

\begin{tabular}{|l|l|l|l|l|l|}
\hline \multirow{2}{*}{ Parameters (mg/I) } & \multicolumn{2}{l}{ North-West } \\
\cline { 2 - 6 } & NW-1 & NW-2 & NW-3 & NW-4 & NW-5 \\
\hline Colour & Colourless & \multicolumn{4}{l|}{} \\
\hline Temperature (oC) & 28.70 & 28.80 & 28.60 & 28.20 & 28.00 \\
\hline pH & 7.90 & 6.40 & 6.70 & 7.10 & 6.20 \\
\hline Acidity & 8.00 & 10.00 & 110.00 & 60.00 & 140.00 \\
\hline Alkalinity & 300.00 & 283.00 & 257.00 & 268.00 & 215.00 \\
\hline Chloride & 72.97 & 66.97 & 97.50 & 69.97 & 64.98 \\
\hline Hardness & 68.00 & 64.00 & 280.00 & 110.00 & 95.00 \\
\hline Sulphate & 55.00 & 48.70 & 63.00 & 30.20 & 19.80 \\
\hline BOD & 0.98 & 1.08 & 0.49 & 0.68 & 0.57 \\
\hline COD & 462.00 & 376.00 & 503.00 & 287.00 & 251.00 \\
\hline TDS (ppm) & 1260.00 & 1200.00 & 1010.00 & 800.00 & 852.70 \\
\hline Lead & & & & & \\
\hline Copper & & & & & \\
\hline Aluminium & & & & & \\
\hline Feal Coifory & & & & \\
\hline
\end{tabular}

Fecal Coliform

Table -8: Physic-chemical characteristic of the water samples

\begin{tabular}{|l|l|l|l|l|l|}
\hline \multirow{2}{*}{ Parameters (mg/l) } & \multicolumn{4}{l}{ South-East } \\
\cline { 2 - 6 } & SE-1 & SE-2 & SE-3 & SE-4 & SE-5 \\
\hline Colour & Colourless & \multicolumn{4}{l}{} \\
\hline Temperature (oC) & 28.00 & 28.20 & 28.70 & 29.00 & 28.10 \\
\hline pH & 6.50 & 7.60 & 7.30 & 7.20 & 7.70 \\
\hline Acidity & 30.23 & 22.89 & 35.21 & 20.84 & 18.71 \\
\hline Alkalinity & 484.00 & 176.57 & 220.83 & 226.90 & 214.01 \\
\hline Chloride & 242.30 & 345.70 & 183.47 & 234.90 & 153.67 \\
\hline Hardness & 282.20 & 156.40 & 202.32 & 181.23 & 150.00 \\
\hline Sulphate & 53.24 & 46.50 & 25.76 & 15.32 & 10.34 \\
\hline BOD & 0.40 & 0.35 & 0.54 & 0.20 & 0.15 \\
\hline
\end{tabular}




\begin{tabular}{|l|l|l|l|l|l|} 
COD & 574.00 & 433.45 & 303.00 & 250.10 & 354.98 \\
\hline TDS (ppm) & 432.00 & 807.00 & 710.00 & 420.00 & 570.00 \\
\hline Lead & & & & & \\
\hline Copper & & & & & \\
\hline Aluminium & & & & & \\
\hline
\end{tabular}

\section{CONCLUSIONS}

Totally fourty one samples taken in and around the dumpsite Valampurivillai, Nagarcoil shows that the levels of dissolved solids, chlorides, hardness are drastically higher than the permissible limits. This poses serious threat to the ground water due to migration of leachate from the solid waste dumping yard.

\section{REFERENCES}

[1] APHA - Standard methods for the examination of water and waste water $-17^{\text {th }}$ Edition 1989.

[2] Arun Jain, Punmia.B.C - Water Supply Engineering, Laxmi Publications Ltd.

[3] HeemanBouwer - Ground Water Hydrology - Taxa McGraw Hill Koga Kusha Ltd.

[4] Kotaiah.B.K and Kumarasamy.N - Environmental Engineering Laboratory Manual - Charotar Publications Ltd.

[5] BIS (Bureau of Indian Standards) 10500, Indian Standard Drinking Water Specification, First Edition 1991, pp 1-8.

[6] Google Maps, Apple Maps. 